\title{
DIMENSION VIA WAITING TIME AND RECURRENCE
}

\author{
Stefano Galatolo
}

\begin{abstract}
Quantitative recurrence indicators are defined by measuring the first entrance time of the orbit of a point $x$ in a decreasing sequence of neighborhoods of another point $y$. It is proved that these recurrence indicators are a.e. greater or equal to the local dimension at $y$. Moreover, the estimation is sharph if some mild assumptions on the statistic of return times are satisfied. These recurrence indicators can hence be used to have an efficient numerical estimation of the local dimension of an invariant measure.
\end{abstract}

\section{Introduction}

The first well known results about recurrence in a dynamical system $(X, T)$ state that under suitable assumptions a typical trajectory of the system comes back infinitely many times in any neighborhood of its starting point. These qualitative results does not give an estimation about the speed of this coming back to the starting point.

A more precise analysis of recurrence was done by defining quantitative recurrence indicators. In the literature such indicators have been defined in several ways by measuring the first return time of an orbit in a decreasing sequence of neighborhoods of the starting point. These sequences of neighborhoods have been defined by the metric of the space $X$, considering a decreasing sequence of balls $([\mathrm{Bo}],[\mathrm{BS}])$ or with respect to the symbolic dynamics induced by a partition, considering a decreasing sequence of cylinders $c_{k}$ on the associated symbolic space $([\mathrm{OW}])$. Other definitions consider the forward images of the whole cylinder $c_{k}$ and consider as a first return for the cylinder the minimum $n$ such that $T^{n}\left(c_{k}\right) \cap c_{k} \neq \emptyset([\mathrm{HSV}],[\mathrm{ACS} 2],[\mathrm{STV}],[\mathrm{BGI}],[\mathrm{CD}])$. In the above cited papers many relations have been then proved between these indicators and other important features of dynamics (for example dimension, entropy, orbit complexity, Lyapunov exponents, mixing properties).

Barreira and Saussol in [BS] prove some relations between quantitative recurrence and the local dimension of an invariant measure. If some technical assumptions are satisfied their recurrence indicator is indeed a.e. equal to the dimension of the invariant measure. Their assumptions are satisfied for example in $C^{1+\alpha}$ systems, with an equilibrum measure supported on a locally maximal hyperbolic set.

Received October 6, 2003. 
In computer simulations or experimental situations, the quantitative recurrence indicators can be easily estimated by looking to the behavior of a "typical, random" orbit and to its first entrance time in a sequence of balls centered in the starting point. By the above results this can give in principle an efficient an efficient numerical estimation of the pointwise dimension of the underlying invariant measure, which is not easy to be known in general. In general systems (where the additional assumptions are not satisfied) however the Barreira and Saussol recurrence indicator gives only a lower bound on the dimension and indeed there are examples were the estimation is not sharph.

We remark that an efficient estimation of the local dimension is important to compute the multifractal spectra that are more and more used in the scientific literature. In the physical literature the recurrence time was already used to investigate some system where the usual box counting tecniques are not efficient (see $[\mathrm{HJ}]$ and the references therein for some examples of computations of local dimension on the Lorenz and Rayleigh-Benard systems). The use of recurrence time however was made on an euristic basis. As we said before there are systems such that the connection between Poincare recurrence and dimension fails. In the present paper we want to provide some rigorous results that can be useful to support an efficient tecnique to calculate local dimension by recurrence and its generalization, the waiting times.

A natural generalization of the quantitative recurrence indicators can be defined by measuring how fast the orbit of a point $x$ approaches near to another point $y$. In the literature about finite alphabet stochastic processes and symbolic systems, indicators of this type were called waiting times. Relations between waiting time and entropy similar the Oerstein Weiss theorem ([OW]) have been proved $([\mathrm{Sh}])$. Such relations hold for Markov chains and in a weaker version in weak Bernoulli processes. Other results relating waiting time with dimension and entropy in the case of finite type shifts with a Gibbs measure or expanding rational maps on the Riemann sphere were given by $[\mathrm{Ch}]$ and $[\mathrm{HV}]$. A recent work $([\mathrm{KS}])$ calculates the waiting time for irrational translations on the circle.

In this work we consider general systems acting on a metric space and we consider a waiting time indicator measuring how much time is needed for the orbit of a point $x$ to enter in a ball centered in $y$. Then we prove some result relating this general indicator to the local dimension (of a measure defined on the space were the dynamics acts).

In $[\mathrm{BGI}]$ and $[\mathrm{DG}]$ a quantitative recurrence argument of this kind was used to estimate the initial condition sensitivity and the orbit complexity of interval exchange transformations and of the Casati-Prosen map (a map arising from poligonal billiards ${ }^{1}$. In the present work this argument is presented in a general setting. The results we present give an upper bound to the local dimension of

\footnotetext{
${ }^{1}$ In these maps a main source of initial conditions sensitivity is given by the fact that nearby starting orbits can be separated by the discontinuities of the map. For this reason the initial condition sensitivity is estimated when we estimate how fast we go near to the discontinuity points.
} 
the measure at each point $y \in X$ via the waiting time indicator (Thm, 4). This is done in several ways giving also weaker results which holds even if the measure $\mu$ is not invariant (Thm. 10,11).

Moreover, if the measure is ergodic and a further tecnical assumption on the statistics of return times is verified the lower waiting time indicator is a.e. equal to the lower local dimension $\underline{d}_{\mu}(x)$ proving that in this case the bound is sharp (Thm. 6). As a partial converse, the bound on the upper dimension can be not sharph in general, as remark 8 shows.

These waiting time indicators are also easy to be estimated numerically by looking to the behavior of a "typical" orbit, measuring its first entrance time in a decreasing sequence of balls centered in $y$. These results and the ones given in [BS] can be combined togheter to have upper and lower bounds on the (upper and lower) local dimension of general systems, thus having a new numerical method to estimate the local dimension in dynamical sytems. One last remark is that most of the following results also hold in systems with an infinite invariant measure.

\section{Main Results}

In the following we will consider a discrete time dynamical system $(X, T)$ were $X$ is a separable metric space equipped with a Borel locally finite measure $\mu$ and $T: X \rightarrow X$ is a measurable map (we remark that we do not assume $\mu(X)=1$ ).

Let us consider the first entrance time of the orbit of $x$ in the ball $B(y, r)$ with center $y$ and radius $r$

$$
\tau_{r}(x, y)=\min \left(\left\{n \in \mathbf{N}, n>0, T^{n}(x) \in B(y, r)\right\}\right) .
$$

By this let us define the quantitative waiting time indicators

$$
\begin{aligned}
& \bar{R}(x, y)=\underset{r \rightarrow 0}{\limsup } \frac{\log \left(\tau_{r}(x, y)\right)}{-\log (r)}=\underset{n \rightarrow \infty}{\limsup } \frac{\log \left(\tau_{2^{-n}}(x, y)\right)}{n} \\
& \underline{R}(x, y)=\liminf _{r \rightarrow 0} \frac{\log \left(\tau_{r}(x, y)\right)}{-\log (r)}=\limsup _{n \rightarrow \infty} \frac{\log \left(\tau_{2^{-n}}(x, y)\right)}{n} .
\end{aligned}
$$

If for some $r \tau_{r}(x, y)$ is infinite then $\bar{R}(x, y)$ and $\underline{R}(x, y)$ are set to be equal to infinity. The indicators $\bar{R}(x)$ and $\underline{R}(x)$ of quantitative recurrence defined in [BS] are obtained as a special case, $\bar{R}(x)=\bar{R}(x, x), \underline{R}(x)=\underline{R}(x, x)$.

We state some first properties of $R(x, y)$. The proof follows directly from the definitions.

Proposition 1. $R(x, y)$ satisfies the following properties

- $\bar{R}(x, y)=\bar{R}(T(x), y), \underline{R}(x, y)=\underline{R}(T(x), y)$.

- If $T$ is Lipschitz, then $\bar{R}(x, y) \geq \bar{R}(x, T(y)), \underline{R}(x, y) \geq \underline{R}(x, T(y))$.

- If $T$ is $\alpha$-Hoelder, then $\bar{R}(x, y) \geq \alpha \bar{R}(x, T(y)), \underline{R}(x, y) \geq \alpha \underline{R}(x, T(y))$. 
Now we are interested to prove relations with dimension. If $X$ is a metric space and $\mu$ is a measure on $X$ the upper local dimension at $x \in X$ is defined as

$$
\bar{d}_{\mu}(x)=\limsup _{r \rightarrow 0} \frac{\log (\mu(B(x, r)))}{\log (r)}=\limsup _{k \in \mathbf{N}, k \rightarrow \infty} \frac{-\log \left(\mu\left(B\left(x, 2^{-k}\right)\right)\right)}{k}
$$

the lower local dimension $\underline{d}_{\mu}(x)$ is defined in an analogous way by replacing limsup with liminf.

In general (even in examples which are interesting in dynamical system theory) $\underline{d}_{\mu}(x)$ and $\bar{d}_{\mu}(x)$ can differ on a positive measure set. If $\bar{d}_{\mu}(x)=\underline{d}_{\mu}(x)=d$ almost everywhere the system is called exact dimensional. In this case many notions of dimension of a measure will coincide (see for example the book $[\mathrm{P}]$ ) and then we have a precise description of the fractal structure of the system. For these and other reasons is important to have estimations for both $\underline{d}_{\mu}(x)$ and $\bar{d}_{\mu}(x)$. With the above notations, Theorem 1 of [BS] can be rewritten as follows

Theorem 2. If $(X, T, \mu)$ is a measure preserving transformation, $\mu(X)=1, X$ is a measurable subset of $\mathbf{R}^{\mathbf{n}}$ then for almost each $x \in X$

$$
\bar{R}(x, x) \leq \bar{d}_{\mu}(x), \underline{R}(x, x) \leq \underline{d}_{\mu}(x) .
$$

For an equilibrium measure supported on a locally maximal hyperbolic set of a $C^{1+\alpha}$ diffeomorphisms [BS] also proved that recurrence and dimension are a.e. equal. The equality also holds in some nonuniformly hyperbolic example, however it is not difficult to see ([BS] example 3$)$ that there are uniquely ergodic irrational rotations $\left(S^{1}, x \rightarrow x+\alpha(\bmod 1)\right)$ such that $\underline{R}(x, x)<\underline{d}_{\mu}(x)$ for each $x \in S^{1}$. In such systems and in general systems $R(x, x)$ then gives only a lower bound for the dimension.

We will see how it is possible to obtain a general upper bound for the dimension in term of $R(x, y)$. In [BGI] is indeed proved

Lemma 3. Let $(X, T)$ be as at the beginning of this section, $\mu$ is an invariant measure, and $y \in X$. If $\alpha>\underline{d}_{\mu}(y)^{-1}$ then for $\mu$-almost all $x \in X$ it holds

$$
\liminf _{n \rightarrow \infty} n^{\alpha} \min _{i \leq n} d\left(y, T^{i} x\right)=\infty .
$$

Here we reformulate and extend this fact in the following way

Theorem 4. If $(X, T)$ is as above, for each fixed $y$

$$
\underline{R}(x, y) \geq \underline{d}_{\mu}(y), \bar{R}(x, y) \geq \bar{d}_{\mu}(y)
$$

holds for $\mu$ almost each $x$.

Proof. First we prove $\underline{R}(x, y) \geq \underline{d}_{\mu}(y)$. We remark that if $\alpha>0$ and $(n+$ $1)^{-\alpha} \leq r \leq(n)^{-\alpha}$, since $\tau_{r}(x, y)$ is decreasing in $r$ then

$$
\frac{\log \left(\tau_{n^{-\alpha}}(x, y)\right)}{-\log \left((n+1)^{-\alpha}\right)} \leq \frac{\log \left(\tau_{r}(x, y)\right)}{-\log (r)} \leq \frac{\log \left(\tau_{(n+1)^{-\alpha}}(x, y)\right)}{-\log \left(n^{-\alpha}\right)}
$$


by this we can see that $\liminf _{r \rightarrow 0} \frac{\log \left(\tau_{r}(x, y)\right)}{-\log (r)}=\liminf _{n \in \mathbf{N}, n \rightarrow \infty} \frac{\log \left(\tau_{n-\alpha}(x, y)\right)}{-\log \left(n^{-\alpha}\right)}$. Now Lemma 3 implies that if $n$ is big enough $\tau_{n^{-\alpha}}(x, y) \geq n$ for each $\alpha>\frac{1}{\underline{d}_{\mu}(y)}$. Then $\liminf _{n \in \mathbf{N}, n \rightarrow \infty} \frac{\log \left(\tau_{n-\alpha}(x, y)\right)}{-\log \left(n^{-\alpha}\right)} \geq \frac{1}{\alpha}$. Since $\alpha$ can be chosen as near as we want to $\frac{1}{\underline{d}_{\mu}(y)}$ we have the statement.

Now we prove $\bar{R}(x, y) \geq \bar{d}_{\mu}(y)$. Suppose $d^{\prime}<\bar{d}_{\mu}(y)$, let us consider

$$
A\left(d^{\prime}, y\right)=\left\{x \in X \mid \bar{R}(x, y)<d^{\prime}\right\} .
$$

By the assumption on the dimension at $y$, if $0<d^{\prime}<d<\bar{d}_{\mu}(y)$ then there is a sequence $n_{k}$ such that

$$
\mu\left(B\left(y, 2^{-n_{k}}\right)\right)<2^{-d n_{k}} \text { for each } k .
$$

On the other side for each $x \in A\left(d^{\prime}, y\right)$ the relation $\tau_{2^{-n}}(x, y)<2^{d^{\prime} n}$ must hold eventually. Let us consider $C(m)=\left\{x \in A\left(d^{\prime}, y\right) \mid \forall n \geq m, \tau_{2^{-n}}(x, y)<2^{d^{\prime} n}\right\}$. This is an increasing sequence of sets "converging" to $A$. If we prove that liminf $\mu(C(m))=0$ the statement is proved. By the definition of $C(m)$ we see $m \rightarrow \infty$ that

$$
C\left(n_{k}\right) \subset \underset{i \leq 2^{d^{\prime} n_{k}}}{\cup} T^{-i}\left(B\left(y, 2^{-n_{k}}\right)\right)
$$

the latter is made of $2^{d^{\prime} n_{k}}$ sets, whose measure can be estimated by Eq. 2, because $T$ is measure preserving. Then $\mu\left(C\left(n_{k}\right)\right) \leq 2^{d^{\prime} n_{k}} * 2^{-d n_{k}}$ and $\mu\left(C\left(n_{k}\right)\right)$ goes to 0 as $k \rightarrow \infty$.

We remark that the above results give an upper bound of the local dimension at each $y \in X$, while the Poincare recurrence based methods works for $\mu$-almost each $x$. This gives the possibility in investigate non typical points. Another interesting possibility given by the use of waiting times for numerical purposes is that for the estimation of the dimension at a certain $y$ one can use different initial points $x_{0}, x_{1}, \ldots$ and improve the convergence speed of the dimension estimator by performing some average of the result over such different initial points (a numerical investigation on this line will appear in $[\mathrm{CG}]$ ).

In the next result we see that under certain hypotesys one of the above inequalities can be reversed.

Definition 5. Let us consider the set

$$
A_{l}(y, r)=\left\{z \in B(y, r): \tau_{r}(z, y) \leq l \mu(B(y, r))^{-1}\right\} .
$$

We say that the system has a reasonable return time statistic at $y$ if there is an $l>0$ such that $\limsup _{r \rightarrow 0} \frac{\mu\left(A_{l}(y, r)\right)}{\mu(B(y, r))}<1$.

The return time statistic is an important and widely studied feature of dynamics. We say that the return time statistics of $(X, T)$ converges to $f$ at the point $y$ if

$$
\lim _{r \rightarrow 0} \frac{\mu\left(\left\{x \in B(y, r), \tau_{r}(x, y) \geq \frac{t}{\mu(B(y, r))}\right\}\right)}{\mu(B(y, r))}=f(t) .
$$


If $f(t)=e^{-t}$ we say that the system has an exponential return time statistic at $y$ (see for example [BSTV] and the references therein for other papers on the subject). If a system has exp. ret. time statistic at some point it has also reasonable return time statistic, moreover this also happen if the statistic converges to any nontrivial $f$. Exponential return time statistic can be found in some systems with uniformly hyperbolic behavior but also in nonuniformly hyperbolic systems like maps on the interval with an indifferent fixed point, complex quadratic maps or in maps of the interval with no dense critical orbits ([BSTV]).

Theorem 6. If $(X, T, \mu)$ is ergodic, the measure is finite, nonatomic and it has a reasonable return time statistic at $y$, then for almost each $x$

$$
\underline{R}(x, y)=\underline{d}_{\mu}(y) .
$$

Proof. $\underline{R}(x, y) \geq \underline{d}_{\mu}(y)$ is already proved in Thm. 4. Now we prove $\underline{R}(x, y) \leq$ $\underline{d}_{\mu}(y)$. Let us consider the set

$$
C=B(y, r)-A_{l}=\left\{x \in B(y, r), \tau_{r}(x, y)>l \mu(B(y, r))^{-1}\right\}
$$

and the sets $T^{-1}(C), T^{-2}(C), \ldots, T^{-l} \operatorname{int}\left(\mu(B(y, r))^{-1}\right)(C)$. All these sets are disjoint because if there was $x \in T^{-i}(C) \cap T^{-j}(C)$ with $i, j \leq l \operatorname{int}\left(\mu(B(y, r))^{-1}\right)$ then $\tau_{r}\left(T^{\min (i, j)}(x), y\right) \leq|i-j|$ and by definition of $C, T^{\min (i, j)}(x)$ cannot be contained in $C$, leading to a contraddition. The set $U_{r}=T^{-1}(C) \cup T^{-2}(C) \cup$ $\ldots \cup T^{-l \operatorname{int}\left(\mu(B(y, r))^{-1}\right)}(C)$ is then such that $\mu\left(U_{r}\right) \geq l \operatorname{int}\left(\mu(B(y, r))^{-1}\right) \mu(C)$. If $y$ has a reasonable statistic of return times then there is a $c>0$ s.t. $\mu(C) \geq$ $c \mu(B(y, r))$ for each $n$ then $\mu\left(U_{r}\right) \geq c \frac{l \operatorname{int}\left(\mu(B(y, r))^{-1}\right)}{\mu(B(y, r))^{-1}}>c^{\prime}>0$.

If $\underline{d}_{\mu}(y)=d$ then for each $\delta>0$ there is a sequence $r_{n} \rightarrow 0$ such that $\mu\left(B\left(y, r_{n}\right)\right) \geq r_{n}{ }^{d-\delta}$. Since $\mu\left(U_{r_{n}}\right) \geq c^{\prime}>0$ we remark that the set of points

$$
G=\left\{x \text { s.t. } x \text { is contained in } U_{r_{n}} \text { for infinitely many } n\right\}
$$

has positive measure.

Finally we remark that if a point $x$ is contained in $U_{r_{n}}$ for some $n$ then $T^{i}(x) \in B\left(y, r_{n}\right)$ for $i \leq l \operatorname{int}\left(\mu\left(B\left(y, r_{n}\right)\right)\right)^{-1}$ and $\mu\left(B\left(y, r_{n}\right)\right) \geq r_{n}{ }^{d-\delta}$. Since $\delta$ is small as wanted and $\frac{-\log \left(\tau_{r_{n}}(x, y)\right)}{\log \left(r_{n}\right)} \leq d+\delta$ is true for infinitely many $n$ then $\underline{R}(x, y) \leq d$. This is true for $x$ belonging in a positive measure set $G$, since $\mu$ is ergodic, then by proposition 1 we have the statement.

Remark 7. With similar tecniques it is possible to prove that if

$$
\liminf _{r \rightarrow 0} \frac{\mu\left(A_{l}(y, r)\right)}{\mu(B(y, r))}<1
$$

then $\underline{R}(x, y) \leq \bar{d}_{\mu}(y)$ then having a lower bound on the dimension even with a considerably weaker assumption on the return time statistic.

In general one cannot expect stronger results like $\bar{R}(x, y)_{\mu} \underset{\bar{a} . e .}{=} \bar{d}_{\mu}(y)$. This is shown in the following examples. 
Remark 8. Let us consider a periodic rotation $\left(S^{1}, x \rightarrow x+\alpha, \lambda\right)$ with $\alpha \in \mathbf{Q}$ and $\lambda$ is the Lesbegue measure, here $\bar{R}(x, y)=\underline{R}(x, y)=\infty$ for each $y$ that is not contained in the orbit of $x$ (that is a finite set) while $\lambda$ has dimension 1. This example is in some sense trivial because the system is not ergodic.

A less trivial example is in a certain sense a small perturbation of this latter one. An irrational $\alpha$ is said to be of type $\nu$ if

$$
\nu=\sup \left\{\beta \mid \liminf _{n \rightarrow \infty} j^{\beta}\left(\min _{n \in \mathbf{N}}|j \alpha-n|=0\right)\right\} .
$$

Lesbegue almost each irrational is of type 1, but there are irrationals with type $>1$. From the main result of $[\mathrm{KS}]$ it can be deduced (with some techical work) that an irrational rotation with angle $\alpha$ of type $\nu>1$ satisfies $\bar{R}(x, y)=\nu>1$ for almost each $x, y$ (while $\underline{R}(x, y) \leq 1$ a.e.).

In the previous results the invariance of the measure was an important ingredient. The following results (where $x$ is fixed and $y$ varies) are more general, they do not require the invariance of $\mu$.

Remark 9. If the measure $\mu$ is not invariant inequality 1 can fail on a positive measure set at some point $y$. For example let us consider a system $(X, T)$, where the map $T$ sends all the space $X$ in a point $y(\forall x, T(x)=y)$ with $\bar{d}_{\mu}(y)>0$. Here $\bar{R}(x, y)<\bar{d}_{\mu}(y)$. We remark that in this example the inequality fails only at one point (y). Next results shows that even when the measure is not preserved the inequality can fail only on a zero measure set (if we fix $x$ and let $y$ vary).

Theorem 10. For each $x \in X$

$$
\bar{R}(x, y) \geq \bar{d}_{\mu}(y), \underline{R}(x, y) \geq \underline{d}_{\mu}(y)
$$

for $\mu$ almost each $y$.

Theorem 11. For each $x \in X$ the set $Y_{h} \subset X$ such that

$$
Y_{h}=\{y \in X, \underline{R}(x, y) \leq h\}
$$

has Hausdorff dimension $\leq h$.

We remark that since obviously $\bar{R}(x, y) \geq \underline{R}(x, y)$ then the above result holds also with $\bar{R}(x, y)$ instead of $\underline{R}(x, y)$.

\section{Proof of Theorems 10 and $\mathbf{1 1}$}

Theorems 10 and 11 come from the following more general results. Let us consider a sequence $x_{i}: \mathbf{N} \rightarrow X$, we define recurrence indicators indicating how the sequence comes near some given points. For this let us consider $y \in X$, and the first entrance time of $x_{i}$ in a ball with center $y$

$$
\tau\left(x_{i}, y, r\right)=\min \left\{n \in \mathbf{N}, n>0, x_{n} \in B(y, r)\right\} .
$$

Let us define the quantitative recurrence indicators 


$$
\begin{aligned}
& \bar{R}\left(x_{i}, y\right)=\limsup _{r \rightarrow 0} \frac{\log \left(\tau\left(x_{i}, y, r\right)\right)}{-\log (r)}=\limsup _{n \rightarrow \infty} \frac{\log \left(\tau\left(x_{i}, y, 2^{-n}\right)\right)}{n} \\
& \underline{R}\left(x_{i}, y\right)=\liminf _{r \rightarrow 0} \frac{\log \left(\tau\left(x_{i}, y, r\right)\right)}{-\log (r)}=\liminf _{n \rightarrow \infty} \frac{\log \left(\tau\left(x_{i}, y, 2^{-n}\right)\right)}{n} .
\end{aligned}
$$

Theorem 11 comes from the following proposition

Proposition 12. For each sequence $x_{i}$ the set $Y_{h} \subset X$ such that $Y_{h}=\{y \in$ $\left.X, \underline{R}\left(x_{i}, y\right) \leq h\right\}$ has Hausdorff dimension $\leq h$.

Proof. We have that $\forall y \in Y_{h} \operatorname{minlim}_{k \rightarrow \infty} \frac{\log \left(\tau\left(x_{i}, y, 2^{-k}\right)\right)}{k} \leq h$. This means that $\forall \epsilon>0, \forall y \in Y_{h}$ and $\forall k_{0} \in \mathbf{N}$ there is $k>k_{0}$ and an index $j$ with $j \leq 2^{(h+\epsilon) k}$ with $y \in B\left(x_{j}, 2^{-k}\right)$.

Let us call $S_{\epsilon, k}$ the union of all the balls $B\left(x_{j}, 2^{-k}\right)$ for all index $j$ such that $j \leq 2^{(h+\epsilon) k}$, that is, $S_{\epsilon, k}=\underset{j \leq 2^{(h+\epsilon) k}}{\cup} B\left(x_{j}, 2^{-k}\right)$. For each $k_{0}$ it then holds $Y_{h} \subset \underset{k \geq k_{0}}{\cup} S_{\epsilon, k}$. By this $Y_{h}$ (and each $S_{\epsilon, k}, k>k_{0}$ ) is covered by a family of balls of diameter less than $2^{-k_{0}}$ and we can estimate the $d$-dimensional Hausdorff measure of $S_{\epsilon, k}$

$$
\mathcal{H}_{2^{-k+1}}^{d}\left(S_{\epsilon, k}\right) \leq 2^{(h+\epsilon) k+1} 2^{(-k+1) d}=2^{k(h+\epsilon-d)+1+d}
$$

and

$$
\mathcal{H}_{2^{-k_{0}}}^{d}\left(Y_{h}\right) \leq \sum_{k \leq k_{0}} 2^{1+d} 2^{k(h+\epsilon-d)}
$$

if $d>h+\epsilon$ we can set $k_{0}$ so big that $\mathcal{H}_{2^{-k_{0}}}^{d}\left(Y_{h}\right) \leq \delta$ for each fixed $\delta$ and $Y_{h}$ is covered by balls of arbitrary small size. This proves $\mathcal{H}^{d}\left(Y_{h}\right)=\lim _{k_{0} \rightarrow \infty} \mathcal{H}_{2^{-k_{0}}}^{d}\left(X_{h}\right)=0$ for each $d>h+\epsilon$. Since $\epsilon$ is arbitrary the statement follows.

Remark 13. By $[\mathrm{BS}]$ (example 3) we have that if $\alpha$ is of type $>1$ (see remark 8) then $\underline{R}(x, x)<1$. By theorem 11 the set of other points $y$ such that $\underline{R}(x, y)=$ $d<1$ is very small, indeed it must have dimension less or equal than $d$.

Theorem 10 comes from

Proposition 14. For $\mu$ almost each $y \in X$

$$
\bar{R}\left(x_{i}, y\right) \geq \bar{d}_{\mu}(y), \underline{R}\left(x_{i}, y\right) \geq \underline{d}_{\mu}(y) .
$$

The proof of proposition 14 is based on the following lemmas

Lemma 15. Let $A=\left\{y \in X, \bar{d}_{\mu}(y)>d\right\}$. If $h<d$ and

$$
Y_{h}=\left\{y \in A \text {, s.t. } \bar{R}\left(x_{i}, y\right)<h\right\}
$$

then $\mu\left(Y_{h}\right)=0$. 
Proof. Let us consider $0<\varepsilon<|h-d|$ and

$$
Y_{h}^{n}=\left\{y \in A \text { s.t. } \forall m>n \tau\left(x_{i}, y, 2^{-m}\right)<2^{(h+\varepsilon) m}\right\} .
$$

We have that $Y_{h} \subset \cup_{n \geq n_{0}} Y_{h}^{n}$ and $Y_{h}^{n} \subset Y_{h}^{n+1}$. If we prove that $\mu\left(Y_{h}^{n}\right)=0$ eventually with respect to $n$ the assertion is proved.

If $y \in Y_{h}^{n}$ then $\forall m>n \exists i$, s.t. $y \in B\left(x_{i}, 2^{-m}\right)$ where $i<2^{m(h+\varepsilon)}$ in other words if we consider the set $\mathcal{B}^{m}$ of all ball of radius $2^{-m}$ with centers $x_{i}$ with $i<2^{m(h+\varepsilon)}$ (that is $\mathcal{B}^{m}=\left\{B\left(x_{i}, 2^{-m}\right)\right.$ s.t. $\left.i<2^{(m(h+\varepsilon))}\right\}$ ) we have that $\forall m>$ $n, Y_{h}^{n} \subset \bigcup_{\beta \in \mathcal{B}^{m}} \beta$.

For each $y \in A$ we have that $\bar{d}(y)=\limsup _{n \rightarrow \infty} \frac{-\log \mu\left(B\left(y, 2^{-n}\right)\right)}{n}>d$, this implies that $\forall y \in A$ there exist an infinite sequence $B\left(y, 2^{-n_{k}}\right)$ of balls centered in $y$ with radius $2^{-n_{k}}$ such that $\mu\left(B\left(y, 2^{-n_{k}}\right)\right) \leq 2^{-n_{k} d}$. Let us call this family of balls $y$-estimated balls.

Now let us consider the balls in $\mathcal{B}^{n}$ for which we have an estimation about their measure: we say that a ball in $\beta \in \mathcal{B}^{n}$ is "nice" if there exist an $y$ such that $\beta$ is contained in some $y$-estimated ball of radius $2^{-n+1}$ found above (we recall that all the balls in $\mathcal{B}^{n}$ have radius $\left.2^{-n}\right)$, thus if $\beta$ is nice then $\mu(\beta) \leq 2^{-(n-1) d}$. Every $y \in Y_{h}^{n}$ has a sequence of $y$-estimated balls, let us consider one of these balls $B\left(y, 2^{-k+1}\right): y$ is also contained in a ball $\beta^{\prime} \in \mathcal{B}^{k}$, then $\beta^{\prime} \subset B\left(y, 2^{-k+1}\right)$. This implies that $\forall j>n$ each point of $Y_{h}^{n}$ is contained in some "nice" ball with radius not greater than $2^{-j}$ that is: $Y_{h}^{n} \subseteq \bigcup_{m>n} \bigcup_{\beta \in\left\{\text { nice balls } \in \mathcal{B}^{m}\right\}} \beta$. Now we can estimate the total measure of the nice balls: we remark that the number of nice balls with radius $2^{-m}$ is not greater than $2^{m(h+\varepsilon)+1}$ and the measure of a nice ball is not greater than the measure of the corresponding $y$ - estimated ball. This implies that $\forall j>n$

$$
\mu\left(Y_{h}^{n}\right) \leq \sum_{m \geq j} 2^{m(h+\varepsilon)+1} 2^{-(m-1) d}
$$

if $n$ is big enough the sum can be set as small as wanted, then $\mu\left(Y_{h}^{n}\right)=0$

Lemma 16. Let $d, c, \delta>0$, let $B=\left\{y \in X, \underline{d}_{\mu}(y)>d+c\right\}$. If $A=\{y \in$ $B$ s.t. $\left.\underline{R}\left(x_{i}, y\right) \leq d-\delta\right\}$, then $\mu(A)=0$.

Proof. Conversely let us suppose that $\mu(A)>0$. Since $\forall x \in A, \underline{d}_{\mu}(x) \geq d+c$ then $x \in A$, implies that if $m$ is big enough (depending on $x$ )

$$
\mu\left(B\left(x, 2^{-m}\right)\right)<2^{-m\left(d+\frac{c}{2}\right)}
$$

then there is an $\bar{m}>0$ and a set $A^{\prime} \subset A$ with $\mu\left(A^{\prime}\right)>0$ such that if $x \in A^{\prime}$ $\forall m>\bar{m} \mu\left(B\left(x, 2^{-m}\right)\right)<2^{-m\left(d+\frac{c}{2}\right)}$ uniformly on all $A^{\prime}$.

By the definition of $A^{\prime}$ for each $k \geq \bar{m}$, each $y \in A^{\prime}$ is contained in some ball $B\left(x_{j}, 2^{-i}\right)$ with $j>k$ and $j<2^{d i}$. 
Now the measure of these balls can be estimated as before using Eq. 5 and then the total measure of $A^{\prime}$ can be estimated as in the previous proof, concluding that $\mu(A)=0$.

Proof of proposition 14. If conversely $\bar{R}\left(x_{i}, y\right)<\bar{d}(y)$ on a set $A^{\prime}$ with $\mu\left(A^{\prime}\right)>0$ we can find a constant $c$ and a set $A^{\prime \prime}, \mu\left(A^{\prime \prime}\right)>0$ such that $\bar{R}\left(x_{i}, y\right)<c<\bar{d}(y)$ on $A^{\prime \prime}$, by lemma 16 we obtain $\mu\left(A^{\prime \prime}\right)=0$. Similarly the other inequality can be obtained

\section{References}

[ACS2] V. Afraimovich, J. R. Chazottes, B. Saussol, Pointwise dimensions for Poincaré recurrences associated with maps and special flows, Disc. Cont. Dyn. Syst. - A 9 (2003), 263-280

[BS] L. Barreira, B. Saussol, Hausdorff dimension of measures via Poincaré recurrence, Commun. Math. Phys., 219 (2001), 443-463.

[BGI] C. Bonanno, S. Isola, S. Galatolo Recurrence and algorithmic information Nonlinearity 17 (2004), 1057-1074.

[Bo] M. D. Boshernitzan, Quantitative recurrence results, Invent. Math. 113 (1993), 617-631

[BSTV] H. Bruin, B. Saussol, S. Troubetzkoy, S. Vaienti Return time statistics via inducing. Ergodic Theory Dynam. Systems 23 (2003), 991-1013.

[CG] T. Carletti, S. Galatolo Numerical estimation of local dimension by Waiting times Work in preparation.

[CD] J. R. Chazottes, F. DurandLocal rates of Poincar recurrence for rotations and weak mixing, to appear in Disc. Cont. Dyn. Sys. A (2003).

[Ch] J. R. ChazottesDimensions and waiting times for Gibbs measures. J. Statist. Phys. 98 (2000), 305-320.

[KS] D. H. Kim, B. K. Seo The waiting time for irrational rotations, Nonlinearity 16, Sept. 2003

[DG] M. D. Esposti, S. Galatolo, Recurrence near given sets and the complexity of the Casati-Prosen map Chaos Solitons Fractals 23 (2005), 1275-1284.

[HJ] T. Halsey, M. JensenHurricanes and Butterflies Nature, 428 (2004), 127-128.

[HV] R. Hill, S. Velani, The ergodic theory of shrinking targets. Invent. Math. 119 (1995) 175-198.

[HSV] M. Hirata, B. Saussol, S. Vaienti, Statistics of return times: a general framework and new applications, Commun. Math. Phys. 206 (1999), 33-55

[OW] D. S. Ornstein, H. Weiss, Entropy and data compression schemes, IEEE Trans. Inf. Th. 39 (1993), 78-83

[P] Y. Pesin Dimension theory in dynamical systems Chicago lectures in Mathematics (1997).

[STV] B. Saussol, S. Troubetzkoy, S. Vaienti, Recurrence, dimensions and Lyapunov exponents, J. Stat. Phys. 106 (2002), 623-634

[Sh] P. C. Shields Waiting times: positive and negative results on the Wyner-Ziv problem. J. Theoret. Probab. 6 (1993), 499-519.

Dipartimento Matematica Applicata, Universitá di Pisa, Italy

E-mail address: galatolo@dm.unipi.it

E-mail address: d80288@ing.unipi.it 